\title{
Microfluidic cell culture systems with integrated sensors for drug screening
}

\author{
Samantha Grist, Linfen Yu, Lukas Chrostowski, Karen C. Cheung* \\ Dept. of Electrical \& Computer Engineering, University of British Columbia, Vancouver, BC, \\ CANADA, V6T $1 Z 4$
}

\begin{abstract}
Cell-based testing is a key step in drug screening for cancer treatments. A microfluidic platform can permit more precise control of the cell culture microenvironment, such as gradients in soluble factors. These small-scale devices also permit tracking of low cell numbers. As a new screening paradigm, a microscale system for integrated cell culture and drug screening promises to provide a simple, scalable tool to apply standardized protocols used in cellular response assays. With the ability to dynamically control the microenvironment, we can create temporally varying drug profiles to mimic physiologically measured profiles. In addition, low levels of oxygen in cancerous tumors have been linked with drug resistance and decreased likelihood of successful treatment and patient survival. Our work also integrates a thin-film oxygen sensor with a microfluidic oxygen gradient generator which will in future allow us to create spatial oxygen gradients and study effects of hypoxia on cell response to drug treatment. In future, this technology promises to improve cell-based validation in the drug discovery process, decreasing the cost and increasing the speed in screening large numbers of compounds.
\end{abstract}

Keywords: microfluidics, drug screening, hypoxia, optical oxygen sensor

\section{INTRODUCTION}

The tissue culture environment can have a dramatic influence on cell behavior. Microscale culture systems can offer several advantages for culturing cells in environments that more closely mimic the natural extracellular environment. Miniaturization permits more precise control of the microenvironment, such as gradients in soluble factors. ${ }^{1,2}$ The smallscale devices also permit tracking of low cell numbers or even individual cells. Microfabrication technology permits the development and large-scale production of identical devices for parallel drug screening. The use of transparent materials allows the integration of these chips onto high-content imaging systems, with advantages in speed, cost, and efficiency. In addition, dynamic and spatial control of soluble factor concentration can be implemented using flow control, and integrated with on-chip sensors.

Drugs developed in conventional, static screening systems may not subsequently have the same activity against solid tumors, contributing perhaps to the high attrition rate in drug development ( $\sim 5 \%$ success rate in oncology, overall clinical success rate $\sim 11 \%){ }^{3}$. Towards the goal of providing more predictive data during drug screening, we have investigated two aspects: how a dynamically modulated drug concentration can affect induction of autophagy, and how an integrated oxygen gradient generator and oxygen sensors can be used to examine effects of hypoxia and drug response. The ability to culture, characterize, image, and screen cells within a single automated, portable platform would permit the identification of the most effective drug treatment for a specific patient using cell-based testing on biopsy samples. This approach will permit screening of many prospective drug compounds in a high-throughput fashion and create more realistic tissue models in vitro than is currently possible.

\subsection{Dynamically modulated chemical environment}

Unlike conventional drug screening methods in which the drug at a specific concentration is applied in batch mode to cell culture wells, we created a continuous, time-varying concentration profile to better mimic physiological conditions. Pharmacokinetic data show that serum concentrations of anticancer agents such as lapatinib peak 4 hours after administration. ${ }^{4}$ Our microfluidic platform features an array of individual chambers inside the microfluidic platform,

\footnotetext{
*kcheung@ece.ubc.ca; phone 1604 827-4114; fax 1604 822-5949
}

Microfluidics, BioMEMS, and Medical Microsystems X, edited by Holger Becker, Bonnie L. Gray, Proc. of SPIE Vol. 8251, 825103 - @ 2012 SPIE · CCC code: 0277-786X/12/\$18 · doi: 10.1117/12.911427 
encapsulating the cells in well-defined volumes on the order of $50 \mathrm{~nL}$, with the ability to dynamically control the microenvironment. This platform can be used to manipulate tens to hundreds of cells, providing the ability to analyze small cell populations and assess the drug sensitivity of samples such as primary cancer cells obtained from needle biopsies of solid tumors. These samples typically yield relatively small numbers of cells. The small scale of our platform can address the risk associated with insufficient cell numbers for analysis. Stereotactic core biopsies obtained with 14G needles typically yield approximately 10,000 cells $^{5}$, while $250 \mathrm{~mL}$ of pleural fluid will yield between $0.25-1 \times 10^{6}$ cells. Mousa et al. ${ }^{6}$ have shown that a digital microfluidic device can be used to quantify estrogen in a $1-\mu \mathrm{L}$ volume microaspirate sample of breast tissue collected with a $30 \mathrm{G}$ needle, which represents three orders of magnitude reduction in the 500-mg sample size required for conventional analysis methods as collected by incisional biopsy.

Autophagy is a cellular process in which proteins and organelles are degraded. In this process, a double-membrane vesicle, an autophagosome, engulfs cytoplasm and organelles. The autophagosome fuses with a lysosome so that its contents are degraded by lysosomal enzymes ${ }^{7-9}$. The protein LC3-II is bound to the membrane of autophagosomes, and can be used as a marker for autophagosomes ${ }^{10}$. Autophagy has been shown to protect cancer cells from environmental or therapeutic stress and can be a cell survival mechanism. However, autophagy is also involved in cancer cell death as a non-apoptotic pathway to eliminate cells ${ }^{11}$. Autophagosome generation and degradation, or autophagic flux, can be elevated in cells stressed by hypoxia or anti-cancer treatments including radiation, angiogenesis inhibitors, and tyrosine kinase inhibitors. Induction of autophagy in breast cancer cells following treatment with some anticancer agents is a survival response, and new therapeutic targets are being developed to block such cytoprotective responses.

Using our microfluidic system, we analyzed tamoxifen (TAM) and rapamycin (RAP) induced autophagy and dosedependent effects on cell viability. We used MCF7 cells transfected with LC3 fused to green fluorescent protein (GFPLC3).

\subsection{Spatial gradient generation and on-chip oxygen sensors - Hypoxia}

Hypoxic tissue areas are regions of tissue with low $\mathrm{pO}_{2}$ values. ${ }^{12}$ Tumor hypoxia can lead to aggressive tumor behavior and resistance to radiation and other cancer treatments. ${ }^{13}{ }^{14}$ Sustained, inadequate supply of oxygen has been linked to tumor cell spreading. This reduced oxygen level can be caused by several factors, including reduced tissue perfusion and increased diffusion distances. Oxygen delivery in solid tumors can also be diminished due to abnormalities in the tumour vascular network. Blood flow within vessels in breast tumours can be chaotic, leading to concurrent and countercurrent flows which give both hypoxia and reperfusion ${ }^{15}$, which may cause the generation of reactive oxygen species and oxidative stress.

Current techniques to measure hypoxia include polarographic systems which use probe electrodes. ${ }^{16,17}$ Such electrodes can consume oxygen. In contrast, optical, luminescence-based oxygen sensors do not consume oxygen during measurement, and are not easily contaminated by the sample. ${ }^{18}$ Sensors containing oxygen-sensitive platinum(II) octaethylporphyrin ketone (PtOEPK) are based on the principle of reversible intensity and excited-state lifetime quenching of the luminescent indicator by molecular oxygen. The intensity quenching can be modeled by the SternVolmer equation,

$$
\frac{I_{0}}{I}=1+K_{S V} p O_{2}
$$

where $\mathrm{pO}_{2}$ is the partial pressure of oxygen, $K_{S V}$ is the Stern-Volmer quenching constant, $I_{0}$ is the excited-state luminescence intensity in the absence of oxygen, and $I$ is the excited state luminescence intensity at the oxygen partial pressure under measurement.

In order to create an on-chip oxygen gradient, our design features two control channels adjacent to the cell culture chamber, separated by thin PDMS barriers. Oxygenated and deoxygenated water are flowed through these control channels, and since PDMS has high gas permeability, the oxygen diffuses through the PDMS barriers into the cell culture environment. Forry and Locascio have used a similar design for microfluidic $\mathrm{CO}_{2}$ control. ${ }^{19}$ The culture media perfusion rate is orders of magnitude lower than the control flowrates. This design would permit the creation of stable gradients in $\mathrm{pO}_{2}$ within the cell culture environment, as well as time-varying gradients to model intermittent hypoxia.

We have integrated dye-based optical oxygen sensors integrated into the microfluidic devices to monitor oxygen concentration. Oxygen concentration within the microchannel will be monitored by phosphorescence intensity using the embedded thin-film sensors at the bottom of the channel. 


\section{EXPERIMENTAL METHODS}

\subsection{Chip microfabrication - drug screening}

The chip was fabricated in polydimethylsiloxane (PDMS) using multilayer soft lithography with the additional intermediate step of plastic replica molding. ${ }^{20}$ PDMS (Sylgard) was cast from the silicon mold master, and then a second cast was made using a two-part polyurethane to form a plastic mold master. After curing at $65^{\circ} \mathrm{C}$ for 30 minutes, the PDMS structures were peeled off the plastic mold master. Then, the two PDMS layers were aligned and placed in an oven overnight to allow diffusion bonding between the two layers to occur. Finally, the microfluidic chips were completed by punching holes through them and bonding them to glass slides using plasma bonding.

\subsection{Cell seeding in microfluidic chambers}

MCF-7 breast tumor cells were kindly provided by the laboratory of Dr. Marcel Bally (British Columbia Cancer Research Centre). Cells were maintained in DMEM medium (Invitrogen) supplemented with $10 \%$ fetal bovine serum (FBS), $100 \mathrm{U} / \mathrm{mL}$ penicillin and $100 \mathrm{U} / \mathrm{mL}$ streptomycin. All cells were cultured in flasks for several days prior to microfluidic experiments. The cell seeding sequence in the microchambers was: (1) An $80 \mu \mathrm{L}$ suspension of $\sim 5 \times 10^{6} / \mathrm{mL}$ MCF-7 cells in a pipette tip was inserted into the inlet and the cells were introduced into the cell chamber under hydrostatic force with valve 1 closed. (2) Cells in the flow channel were flushed out and infused with fresh medium with valve 2 closed. (3) After cell loading, the chips were returned to the incubator overnight to permit cell attachment. The microfabricated devices were placed into standard well plates after seeding with cells, and the well plate was placed into an incubator $\left(5 \% \mathrm{CO}_{2}, 37^{\circ} \mathrm{C}\right)$. The microfluidic chips were connected to a syringe pump (Cole Parmer) and DMEM culture medium was circulated.
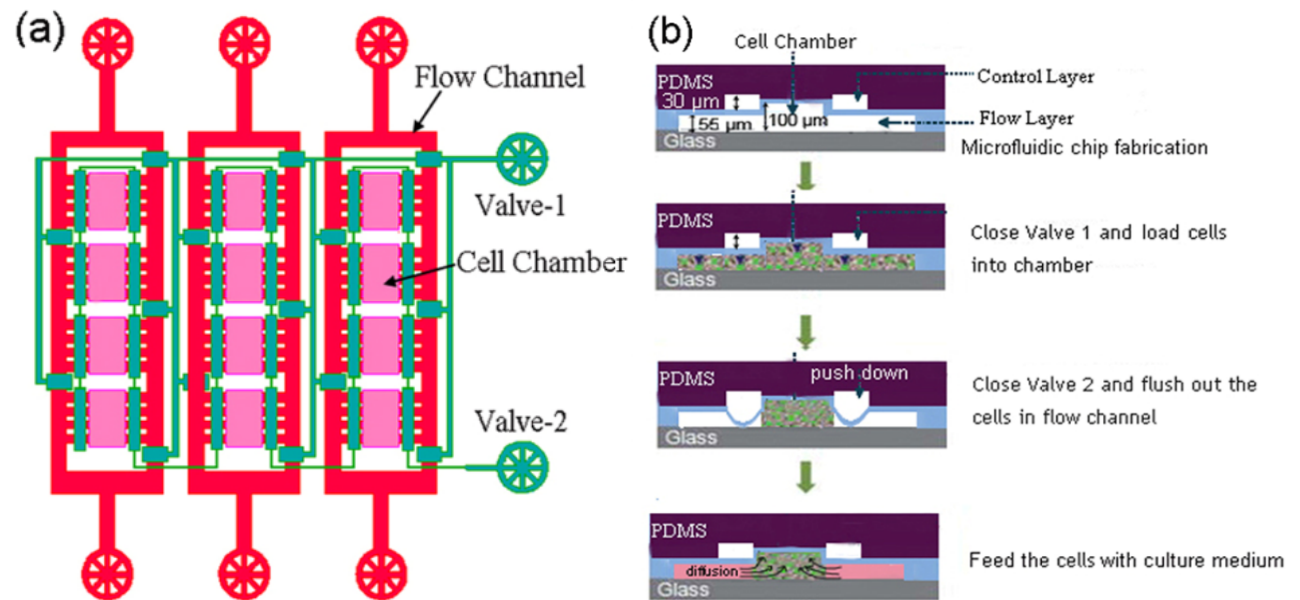

Figure 1. Schematic diagram of multi-layer microfluidic cell culture chip. (a) Layout of the microfluidic device composed of two layers including cell chamber \& flow layer and control layer. There are $4 \times 3$ cell culture chambers in each single chip. Each chamber was $600 \mu \mathrm{m}$ in width, $100 \mu \mathrm{m}$ in height, $1000 \mu \mathrm{m}$ in length. (b) Cell loading steps.

\subsection{Drug treatment}

Autophagosome formation was induced by loading a time-varying concentration of RAP and TAM for 72 hours using syringe pumps. The drug concentration curve was modeled in two parts: In the first 6 hours, the concentration $\mathrm{y}_{1}$ was calculated according to: $\mathrm{y}_{1}=\left(\mathrm{C}_{0} \times \mathrm{V}_{0}+\mathrm{v}_{1} \times \mathrm{t} \times \mathrm{C}_{\mathrm{i}}\right) /\left(\mathrm{V}_{0}+\mathrm{v}_{1} \times \mathrm{t}\right)$; between 6 to 24 hours, the concentration $\mathrm{y}_{2}$ was calculated according to: $\mathrm{y}_{2}=\left[\left(\mathrm{V}_{0}+\mathrm{v}_{1} \times 360\right) \times \mathrm{C}_{\mathrm{f}}\right] /\left[\left(\mathrm{V}_{0}+\mathrm{v} 1 \times 360+v 2 \times(\mathrm{t}-360)\right]\right.$, where $\mathrm{t}, \mathrm{C}_{0}, \mathrm{C}_{\mathrm{i}}, \mathrm{C}_{\mathrm{f}}, \mathrm{V}_{0}, \mathrm{v}_{1}, \mathrm{v}_{2}$, are time, original concentration, injected high concentration, final concentration, original volume, the flow rate of injected high concentration of drug during the first 6 hours, the flow rate of culture medium during the following 18 hours. Here, $\mathrm{C}_{0}$, $\mathrm{C}_{\mathrm{i}}, \mathrm{C}_{\mathrm{f}}$ were set at $2.5 \mu \mathrm{M}, 100 \mu \mathrm{M}$ and $20 \mu \mathrm{M}$ respectively. $\mathrm{V}_{0}, v_{1}, v_{2}$ were set at $5760 \mu \mathrm{L}, 3.5 \mu \mathrm{L} / \mathrm{min}$, and $45.5 \mu \mathrm{L} / \mathrm{min}$ respectively. This profile was repeated for 3 days.

As a control, cells were treated at constant concentrations of tamoxifen and rapamycin, at concentrations of $0.5,2.5,5$, 10 , and $20 \mu \mathrm{M}$ for 72 hours. 


\subsection{Assessment of cell viability and induction of autophagy}

The cells were stained and analysed by 3 color scanning laser confocal fluorescence microscopy (Olympus). First, ethidium homodimer-1 (EthD-1) (Invitrogen), which is excluded by the intact plasma membrane of live cells, was used to stain for dead cells. A solution of $4 \mu \mathrm{M}$ EthD-1 was injected into the channel with a syringe and incubated for $45 \mathrm{~min}$. The cells were then fixed in $4 \%$ paraformaldehyde, then stained with 4,6-diamidino-2-phenylindole (DAPI) as an indicator for the total number of cells. Cells were considered non-viable if they showed overlapping staining for EthD-1 and DAPI.

Under conditions which trigger autophagy, GFP-LC3 relocates to the membrane of newly formed autophagosomes which appear as green fluorescent puncta in the cellular cytoplasm. ${ }^{21}$ Each LC3-GFP puncta, or spot, is one autophagosome. To quantify the induction of LC3 aggregation, cells were separated into two groups of $\leq 5$ versus $>5$ GFP-puncta per cell.

\subsection{Simulation of oxygen concentration gradients}

Estimated oxygen concentration gradient profiles were obtained by 3-D, steady-state simulations in COMSOL $\mathbb{R}$ Multiphysics. For these simulations, a spatially and temporally constant temperature of $25^{\circ} \mathrm{C}$ was used to match current experimental conditions. The simulated geometry contained a $(750 \mu \mathrm{m}$ wide) main cell culture channel as well as two additional 'oxygen control' channels ( $300 \mu \mathrm{m}$ wide, one on either side of the cell culture environment) with a $100 \mu \mathrm{m}$ PDMS layer between these channels and the cell culture environment, permitting oxygen diffusion through the PDMS membrane into the cell culture environment.

The design was modeled with a $100 \mu \mathrm{m}$ channel depth, and the flow simulations used the COMSOL ${ }^{\circledR}$ Incompressible Navier-Stokes model. All initial velocities in the media were set to zero, no slip boundary conditions were assumed for all walls, and mesh independence was ensured. Oxygenated and deoxygenated water were simulated to flow through the control channels at a much higher flow rate than the rate of cell culture media perfusion (an input/output pressure difference of $400 \mathrm{~Pa}$ was simulated, resulting in an average flow rate of $100 \mu \mathrm{L} / \mathrm{min}$, while the cell culture media perfusion rate was simulated as $0.3 \mu \mathrm{L} / \mathrm{min}$ consistent with prior studies by our group ${ }^{22}$ ). The perfusion media and the environment around the PDMS were simulated as being deoxygenated.

The oxygen diffusion simulations used the COMSOL ${ }^{\circledR}$ Convection and Diffusion model and were coupled to the flow simulations via the media flow velocities. The diffusion constants used for the materials of interest are: $3.4 \cdot 10^{-9} \mathrm{~m}^{2} / \mathrm{s}$ for PDMS at $35^{\circ} \mathrm{C}^{.23}$ and $2.2 \cdot 10^{-9} \mathrm{~m}^{2} / \mathrm{s}$ for water at $25^{\circ} \mathrm{C} .^{24}$

The boundaries between the PDMS and the channels were set as continuity (permitting diffusion), and the boundary at the bottom of the channels was set as insulating to approximate the minimal oxygen diffusion through glass. The initial value of the oxygen level in the channels and PDMS was set to zero (deoxygenated). The boundaries at the two oxygen control inlets were set as concentration boundary conditions with values for oxygen-saturated and deoxygenated water, and the two outlets were set as convective flux boundary conditions.

The deoxygenated water was assumed to have an oxygen concentration of $0 \mathrm{mols} / \mathrm{m}^{3}$. To obtain the oxygen concentrations for the oxygen-saturated water, an equation proposed using thermodynamic theory by Tromans ${ }^{25}$ was used, yielding a molarity for oxygen-saturated water at $1 \mathrm{~atm}$ and $25^{\circ} \mathrm{C}$ of $1.26 \mathrm{mols} / \mathrm{m}^{3}$; this value was used as the concentration boundary condition at the oxygenated inlet of the device. All outer PDMS boundaries were set as concentration boundary conditions, with zero oxygen.

\subsection{Microfluidic chip and oxygen sensor fabrication}

The microfluidic gradient generator chips were also fabricated using poly(dimethylsiloxane) using a standard soft lithography process employing a single-layer SU-8 mold fabricated using photolithography, and inlet and outlet holes were punched using needle tips. The optical oxygen sensors were kindly fabricated by Dr. V. Nock at the University of Canterbury using the fabrication process described in his 2010 paper. ${ }^{26}$ Briefly, the oxygen-sensitive phosphor platinum octaethylporphyrin ketone (PtOEPK) was dissolved in a 7\% solution of polystyrene (PS) in toluene at a concentration of $1 \mathrm{mg} / \mathrm{mL}$ and spun onto glass microscope slides, evaporating the toluene to leave a thin ( $\sim 1 \mu \mathrm{m}$ thick $)$ PtOEPK-PS film. This film was then patterned using photolithography through a sacrificial metal layer (as the photoresist strip step would also attack the PtOEPK-PS layer if no sacrificial metal were used).

After sensor patterning, the PDMS chips were aligned over the patterned sensors and reversibly bonded. The chips were held in a jig during the experiments to prevent leakage. 


\subsection{Experimental setup for oxygen gradient generation and measurement}

Preliminary oxygen gradient measurements were obtained by supplying oxygen-saturated water to one oxygen control channel and deoxygenated water to the other. The measurement setup for these measurements is presented in Fig. 2. Nitrogen (to deoxygenate) and oxygen (to oxygenate) (Praxair) were bubbled through filtered water using Pyrex fritted gas dispersion tubes (Fisher Scientific \#11-138B). Oxygen-impermeable Saran ${ }^{\circledR}$ tubing (Pickering Laboratories) was used to carry the oxygen control fluids from the bubblers to the microfluidic chip, and a syringe pump (KD Scientific) using negative pressure was employed to flow the fluids through the control channels at a flow rate of $100 \mu \mathrm{L} / \mathrm{min}$. A second identical syringe pump was used (again under negative pressure) to flow filtered water through the microfluidic chip's main channel at a rate of $0.3 \mu \mathrm{L} / \mathrm{min}$, simulating media perfusion.

The oxygen sensors were imaged using a Nikon TE-2000U inverted fluorescence microscope with custom filter block (Omega Optical) and high intensity mercury phosphorescence excitation source. The reference deoxygenated $\left(\mathrm{I}_{0}\right)$ images were acquired while deoxygenated water was supplied to both control channels (again at a rate of $100 \mu \mathrm{L} / \mathrm{min}$ ) and the chip was exposed to an $\mathrm{N}_{2}$ atmosphere. In all cases, three images acquired over a time period of approximately $20 \mathrm{~s}$ were averaged, and 300 pixel rows were averaged to obtain each intensity cross-section. The acquired images were imported into MATLAB ${ }^{\circledR}$ and analyzed using custom MATLAB ${ }^{\circledR}$ scripts.

For the purposes of comparing the experimental intensity results with those from simulation, the experimental oxygen concentrations were approximated using Equation 1. Previous fluidic calibration of the sensors indicated that their SternVolmer quenching constants $\left(\mathrm{K}_{\mathrm{SV}}\right)$ were approximately $6.2 \mathrm{M}^{-1}$.

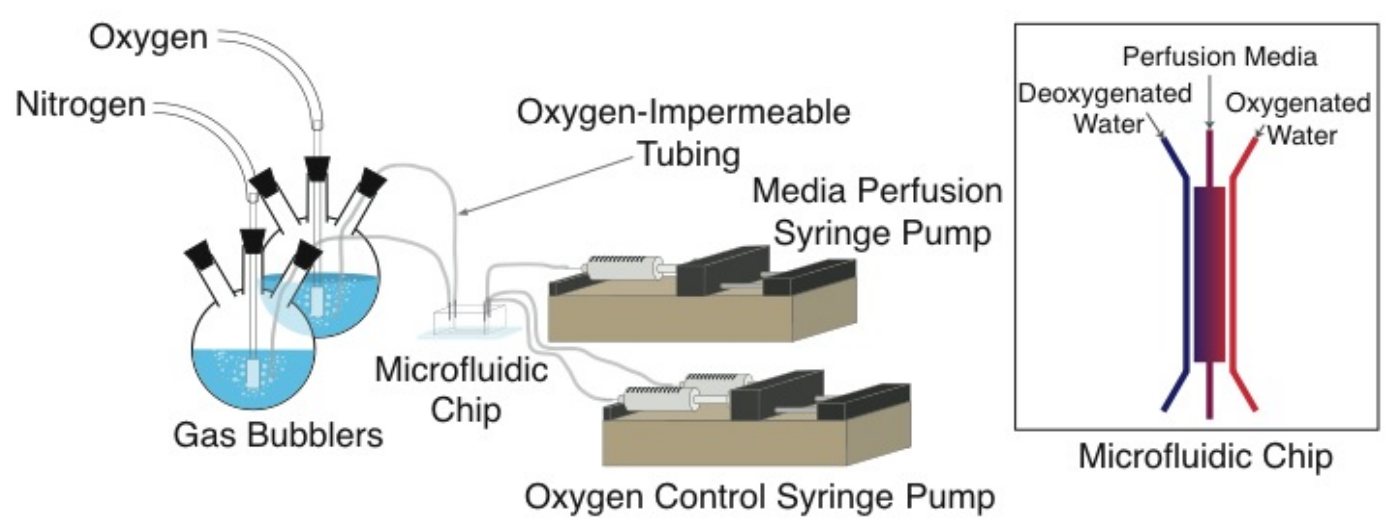

Figure 2. Experimental setup for oxygen gradient generation measurements. The inset depicts the fluids and relative oxygen levels supplied to the microfluidic chip.

\section{RESULTS AND DISCUSSION}

\subsection{On-chip culture}

After 24 hours culture, cells in microchambers showed good adhesion and morphology (Fig. 3).
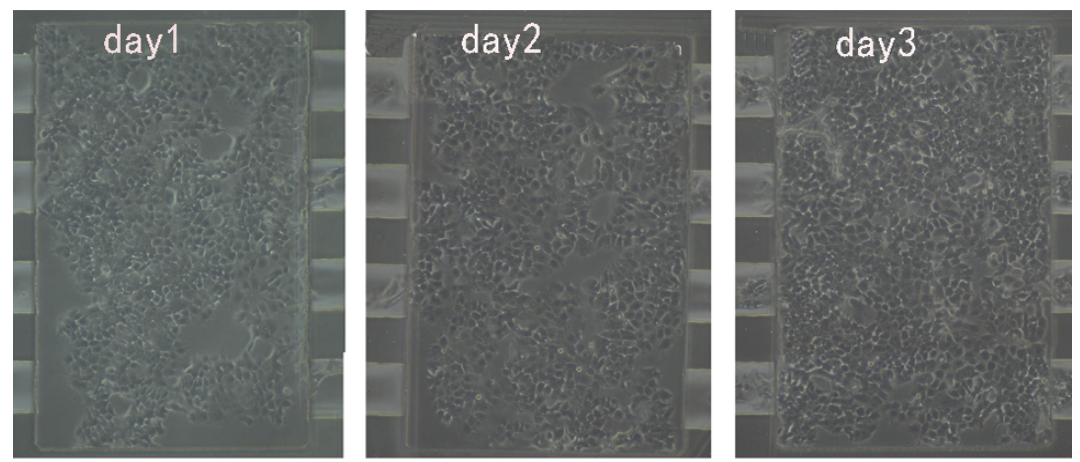

Figure 3. Representative micro-photographs showing cells having good adhesion and morphology. 


\subsection{Time-varying drug profile}

The time-varying concentration profile treatment was compared with non-varying constant concentration treatments. The time-varying concentration profile created in the chip was verified using fluorescein isothiocyanate (FITC) and fluorescence intensity measurement (Fig. 4). In the first 6 hours, drug concentration increased from a minimum of $2.5 \mu \mathrm{M}$ to a maximum of $20 \mu \mathrm{M}$, and then decreased to $2.5 \mu \mathrm{M}$ in the following 18 hours. According to the calculated area under the curve, the average concentration over this time period was $10.48 \mu \mathrm{M}$.

Cells showed diffuse distribution of green fluorescence in the absence of drug; the number of LC3-GFP-positive autophagosomes is low during control conditions. Following treatment with tamoxifen and rapamycin, the MCF-7 cells showed an increase in punctate localization of GFP-LC3.

Both tamoxifen and rapamycin are known to activate autophagy. ${ }^{21,}{ }^{27}$ Tamoxifen is used to treat estrogen-receptor positive $(\mathrm{ER}+)$ cancers, and it has been shown to induce autophagy in breast cancer cells ${ }^{28}$. Treatment with tamoxifen increased the number of LC3-GFP spots and their fluorescence intensity (Fig. 5). In $2.5 \mu \mathrm{M}$ treated samples, the number of cells with more than 5 puncta increased from $6 \%$ in controls to $16 \%$, and to $18 \%$ in $5 \mu \mathrm{M}$ treated samples. This is similar to the findings of Qadir et al. ${ }^{28}$ who used the same cells in conventional well plates for culture and tamoxifen treatment.
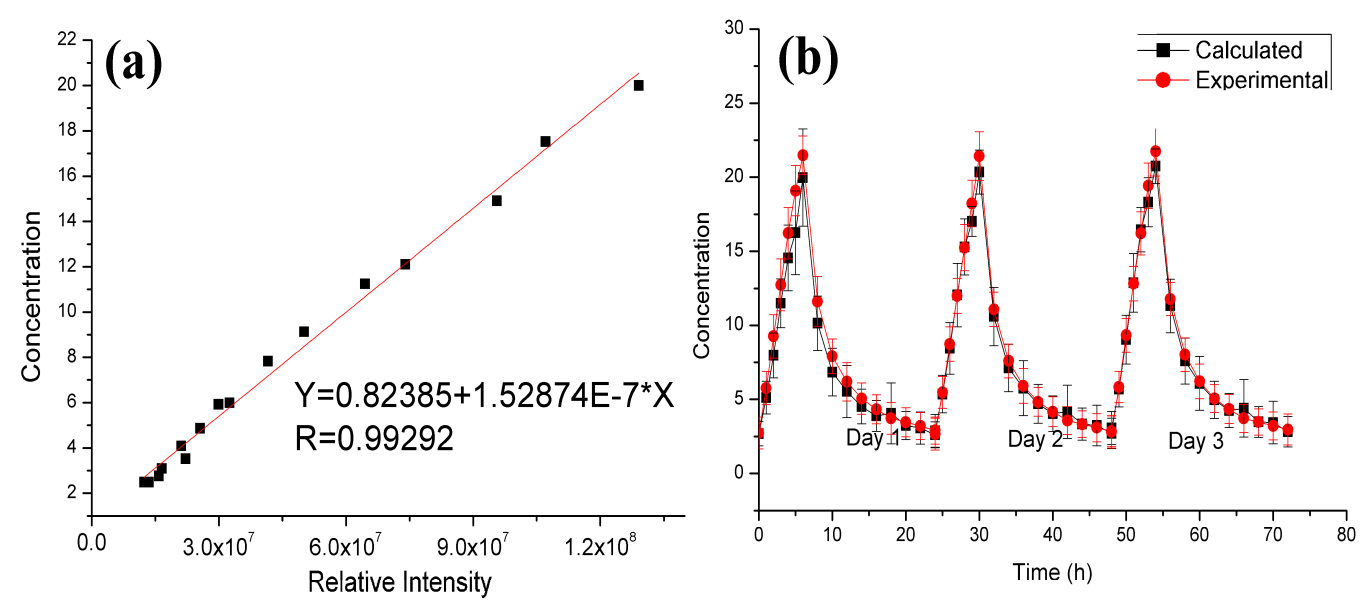

Figure 4. Time-varying concentration profile as verified using fluorescence intensity measurement. (a) The relationship between the FITC concentration and relative fluorescent intensity. (b) The calculated FITC concentration compared to experimental FITC concentration in order to simulate the real drug concentration curve. The calculated area under the peak in the experimetal curve is 251.5025 , which is equal to an average concentration of $10.48 \mu \mathrm{M}$.

Fig. 6 shows that cell viability decreased after treatment with time-varying concentration compared to that of constant concentration of $10 \mu \mathrm{M}$. In constant $10 \mu \mathrm{M}$ tamoxifen treated samples, the viability was $75 \%$ after three days, whereas the time-varying profile treated samples had $35 \%$ viability. The calculated area under the curve of the time-varying concentration profile gives an average of $10.48 \mu \mathrm{M}$ over this time period. The lower viability indicates that cells are more sensitive to the time varying concentration tamoxifen treatment which moves from a minimum of $2.5 \mu \mathrm{M}$ to a maximum of $20 \mu \mathrm{M}$.

Although a constant $10.48 \mu \mathrm{M}$ treatment and the time-varying treatment would give the same total drug exposure, the time-varying profile peaked at $20 \mu \mathrm{M}$, and this may be one reason for the reduced viability.

Fig. 7 shows the percentage of MCF7 cells showing LC3 aggregation after treatment with time-varying concentration of TAM was comparatively lower than that of cells treated with constant concentration of 10 and $20 \mu \mathrm{M}$ drugs for 3 days. In the samples treated at a constant concentration of $10 \mu \mathrm{M}$ tamoxifen over 3 days, the number of cells with more than 5 puncta increased to $30 \%$, while the $20 \mu \mathrm{M}$ treated samples increased to $80 \%$. In contrast, however, the cells treated with the time-varying tamoxifen profile showed an increase to only $22 \%$. 


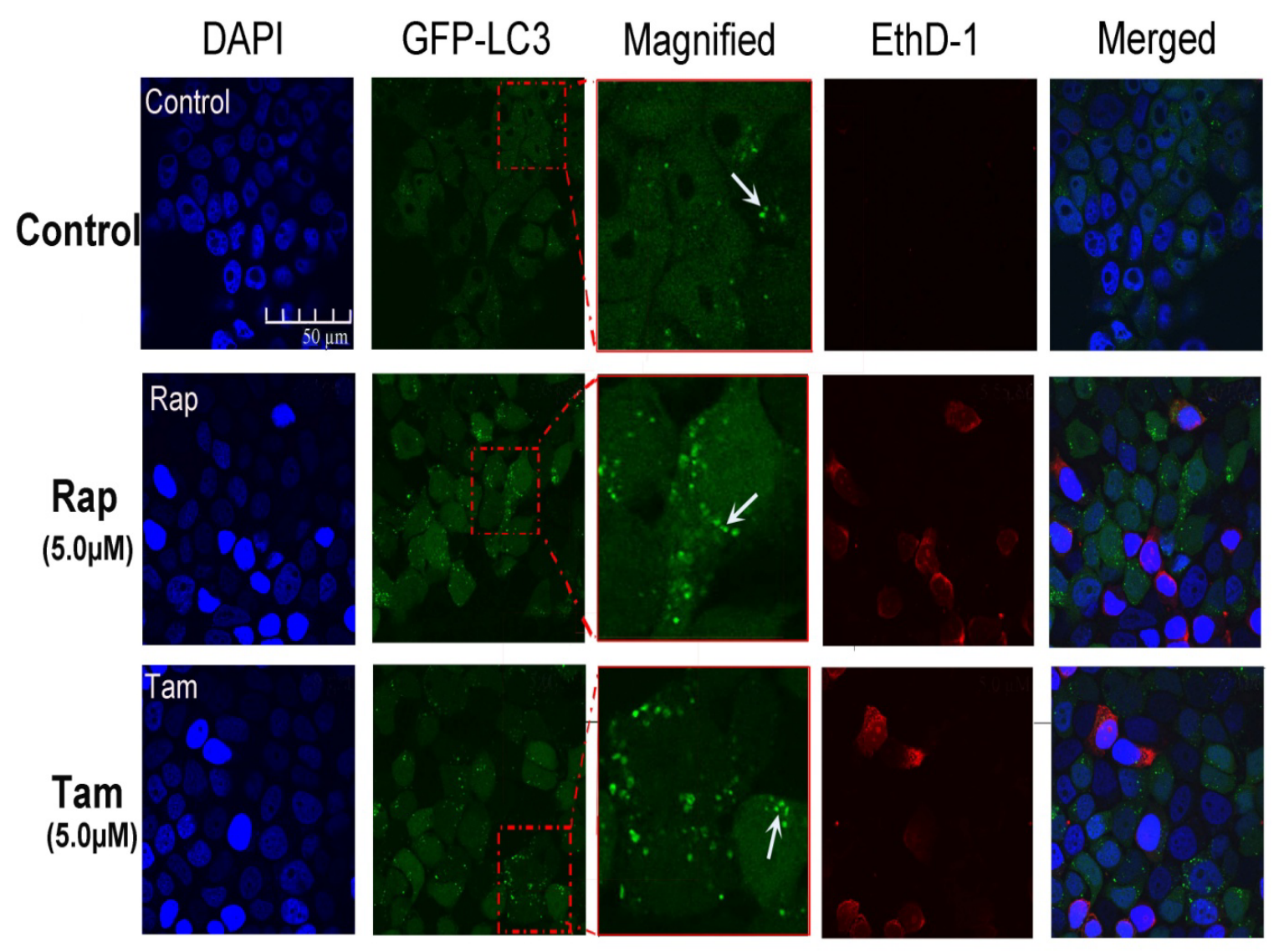

Figure 5. Three color fluorescent images of untreated and treated MCF7 cells (DAPI: total cell count; EthD-1: dead cells) $(5 \mu \mathrm{M}$ constant concentrations of RAP and TAM over 72 hours). Treated cells show an increased punctate pattern of GFP-LC3 expression compared to the control. An arrowhead shows the localization of GFPLC3 puncta, indicative of autophagosomes.
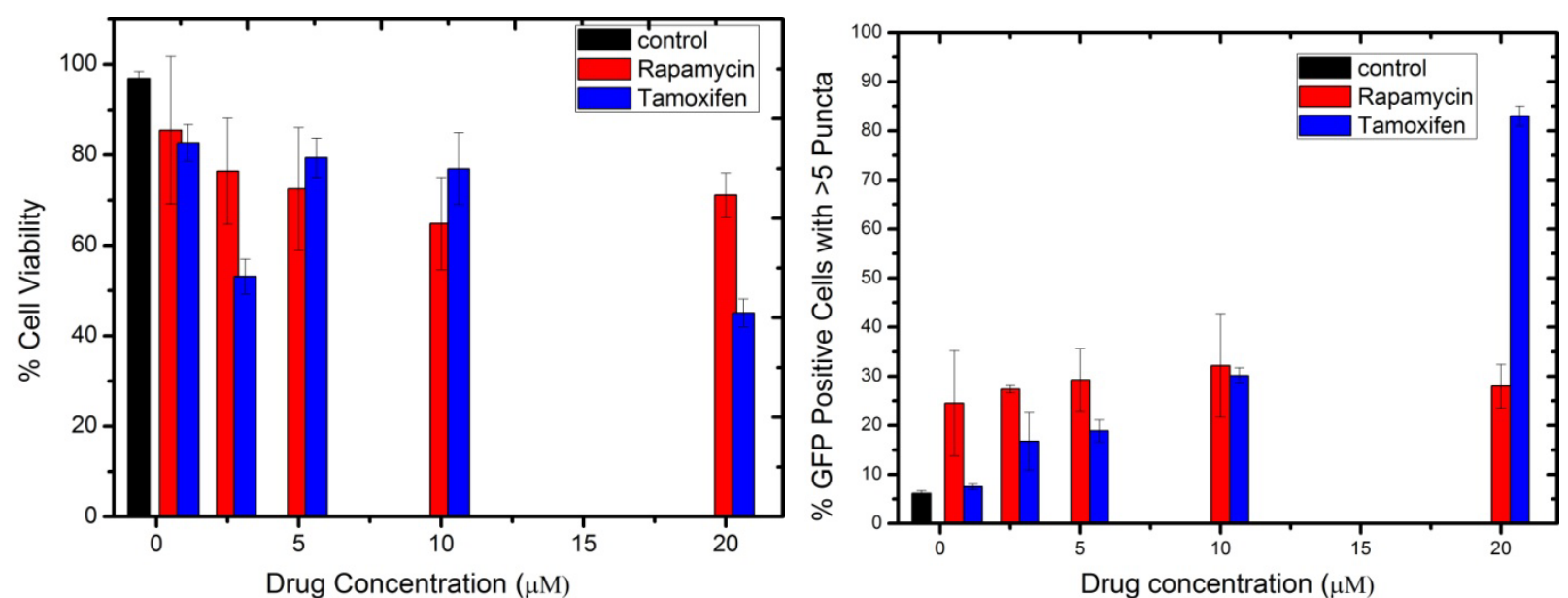

Figure 6. Effects of different constant concentrations of rapamycin and tamoxifen on cell viability and autophagy after 72 hours treatment using constant drug concentrations. (a) At high drug concentration, the TAM treated cells have much lower viability than RAP treated cells. (b) About $83 \%$ of MCF7 cells treated with $20 \mu \mathrm{M}$ TAM for 3 days showed LC3 aggregation, whereas these autophagic features were detected only in $7.5 \%$ in $0.5 \mu \mathrm{M}$ TAM treated cells. 


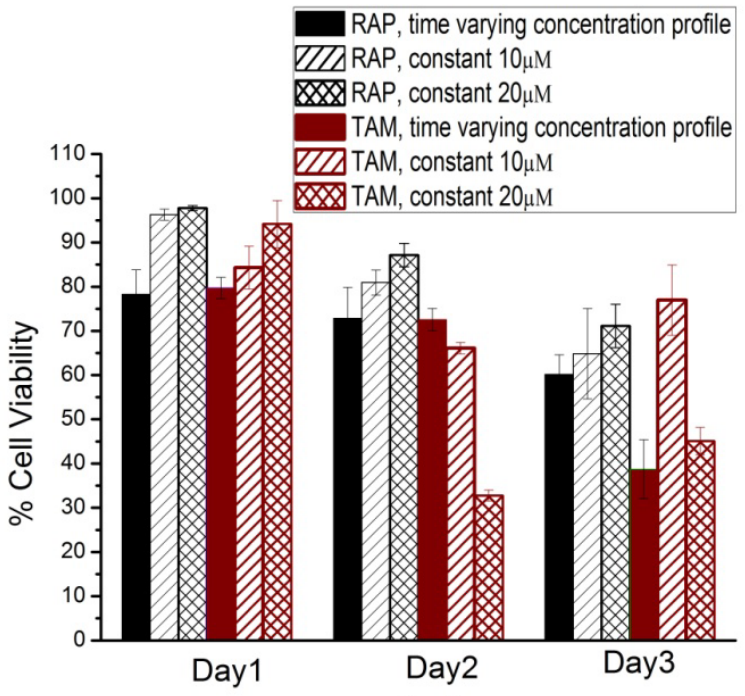

(a)

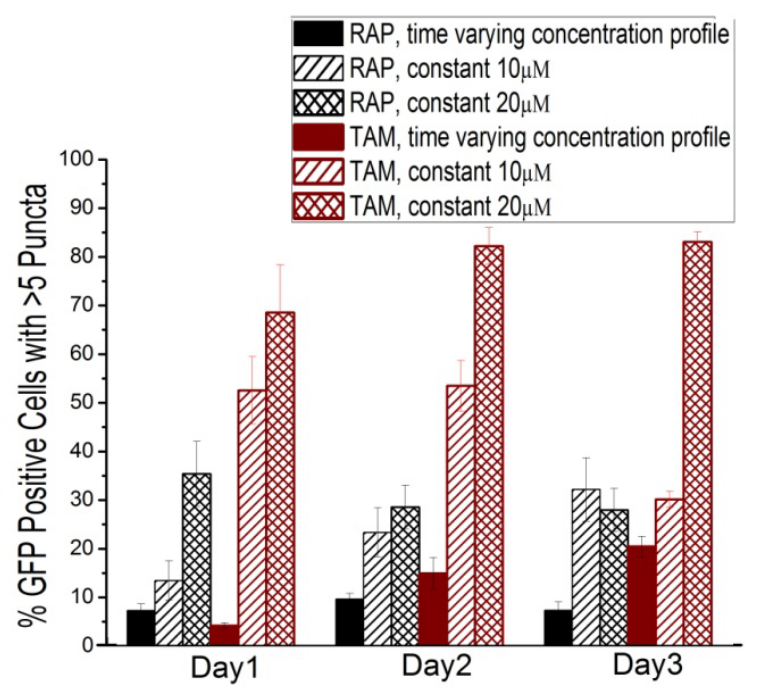

(b)

Figure 7. This figure shows the effects of rapamycin and tamoxifen on (a) cell viability and (b) autophagy. One group of cells was treated with time varying gradient concentration of drugs for 1, 2 and 3 days respectively. The drug concentration varied with time as shown in Fig. 4. Another two groups of cells were treated with constant concentration of $10 \mu \mathrm{M}$ and $20 \mu \mathrm{M}$ RAP and TAM for 1, 2 and 3 days respectively. The cell viability of gradient concentration drug treated cells was lower than that of constant concentration of 10 and $20 \mu \mathrm{M}$ drugs treated cells at the first and third days.

The percentage of dead cells with GFP puncta was much lower than that of cells only showing with $>5$ puncta, indicating that cells with more autophagic features had higher viability at lower drug concentrations. Thus, at lower drug concentrations, autophagy may be reversible and a defensive mechanism to protect tumor cells from cell death. High drug concentrations may trigger irreversible autophagic processes that induced programmed cell death.

\subsection{Oxygen gradient: simulation}

The results of these simulations are presented in Fig. 8, with the simulated design shown at the top left of the image. Within the image, there are 6 line plots, showing the different cross-section positions and channel materials. All crosssections were taken at the bottom of the channel, to indicate the eventual reading of oxygen sensor films integrated into the channel bottom.

Fig. 8 shows that it is possible to control the oxygen concentration gradient across the cell culture area using the oxygen control channels. The oxygen control may be significantly improved by setting the outside walls of the PDMS to be impermeable to oxygen; this should be possible by coating the outside of the PDMS block (e.g. with Parylene ${ }^{29}$ ). The critical oxygen partial pressure $\left(\mathrm{pO}_{2}\right)$ value in solid tumor tissue is generally defined to range from $5-10 \mathrm{~mm} \mathrm{Hg}^{30}$ or 8$10 \mathrm{~mm} \mathrm{Hg}^{14}$. Again using thermodynamic theory by Tromans ${ }^{25}$, the approximate concentration values in solid tumor tissue (for a $\mathrm{pO}_{2}$ of $10 \mathrm{~mm} \mathrm{Hg}$ ) should be $5 \cdot 10^{-5} \mathrm{M}$; it should be possible to reach this region of concentrations in this gradient generator design by lowering the oxygenated input concentration. 


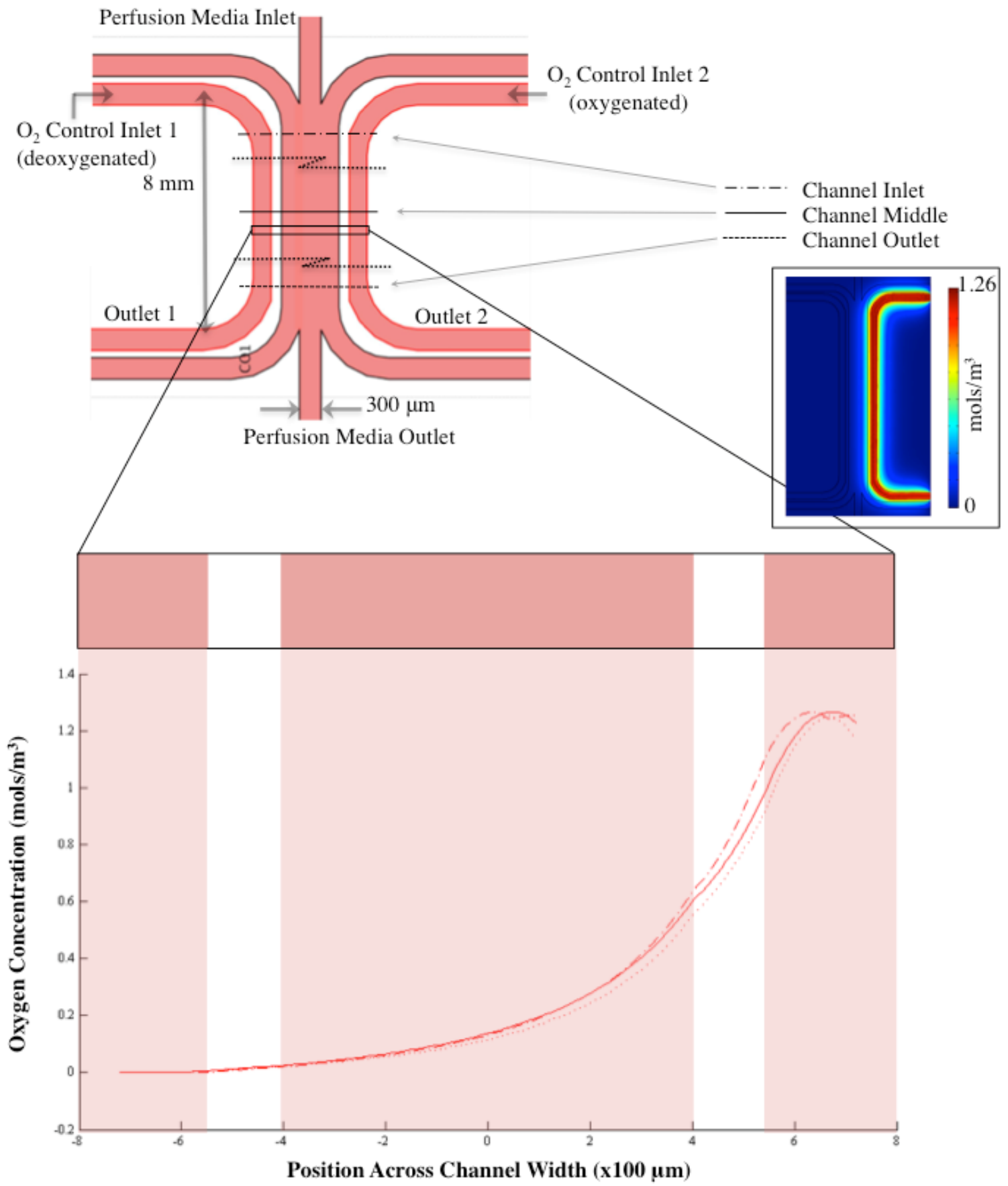

Figure 8. COMSOL ${ }^{\circledR}$ simulated oxygen concentration gradients across channel cross-sections at differing locations down the length of the channel, for the microfluidic oxygen gradient generator design. The line styles indicate the cross-section position in the channel (dash-dot for inlet, solid for middle, and dot for outlet). The inset shows a color map of the oxygen levels at the bottom of the channel.

\subsection{On-chip creation of oxygen gradient}

The flows in the control channels were able to create a gradient in the oxygen level across the main channel. Figure 9 depicts the measured and simulated oxygen concentration gradients for the inlet, midpoint, and output of the gradient generator channel. It is evident that the range of oxygen concentrations created and measured experimentally in the 
device is not currently as large as those predicted; we expect that this is due to oxygen leakage in our system as well as optical factors in our measurement setup and we are currently modifying the setup to overcome these challenges.

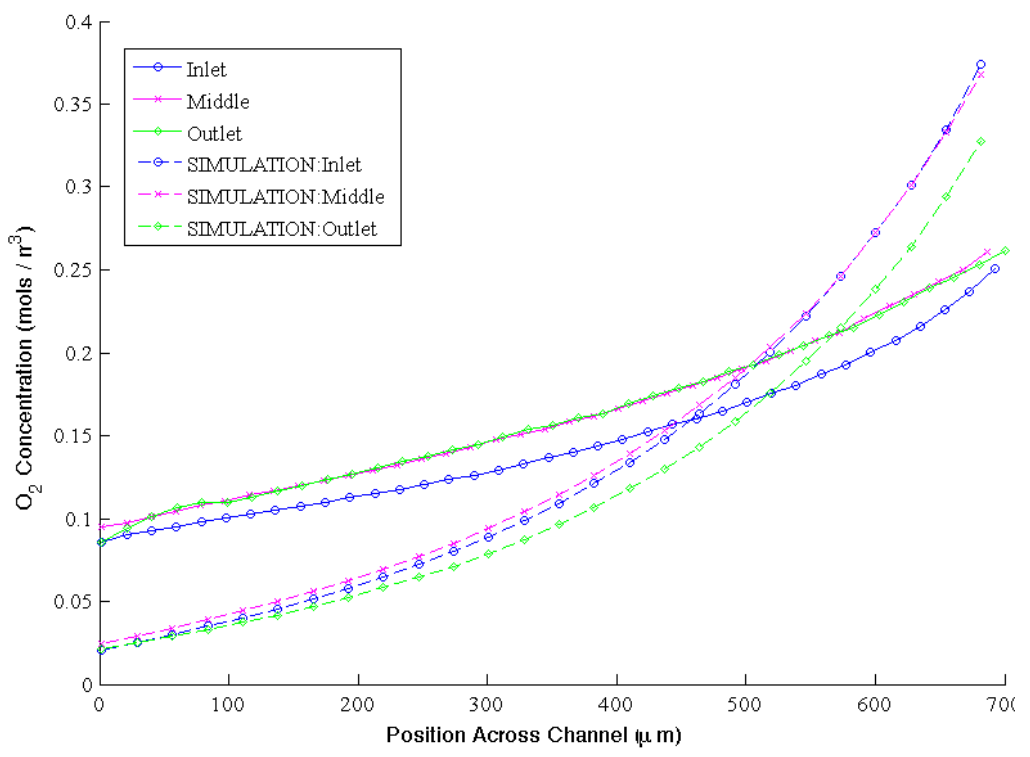

Figure 9. Comparison of experimental and simulated oxygen concentration gradients for three different locations in the microfluidic oxygen gradient generator.

\subsection{Simulation oxygen gradient - model of hypoxia}

Figure 10 shows another COMSOL ${ }^{\circledR}$ simulation model, this time assuming that the outside of the PDMS block containing the channels is coated to render it impermeable to oxygen (for example by coating the device with parylene). In addition, instead of supplying oxygen-saturated water to the fluidic inlet, this simulation used the solid tumor oxygen level of $10 \mathrm{~mm} \mathrm{Hg}$ as the input concentration to the oxygenated inlet. Figure 10 suggests that it is possible to produce a linearly-varying spatial oxygen concentration gradient in the hypoxic range of interest using the gradient generator design.

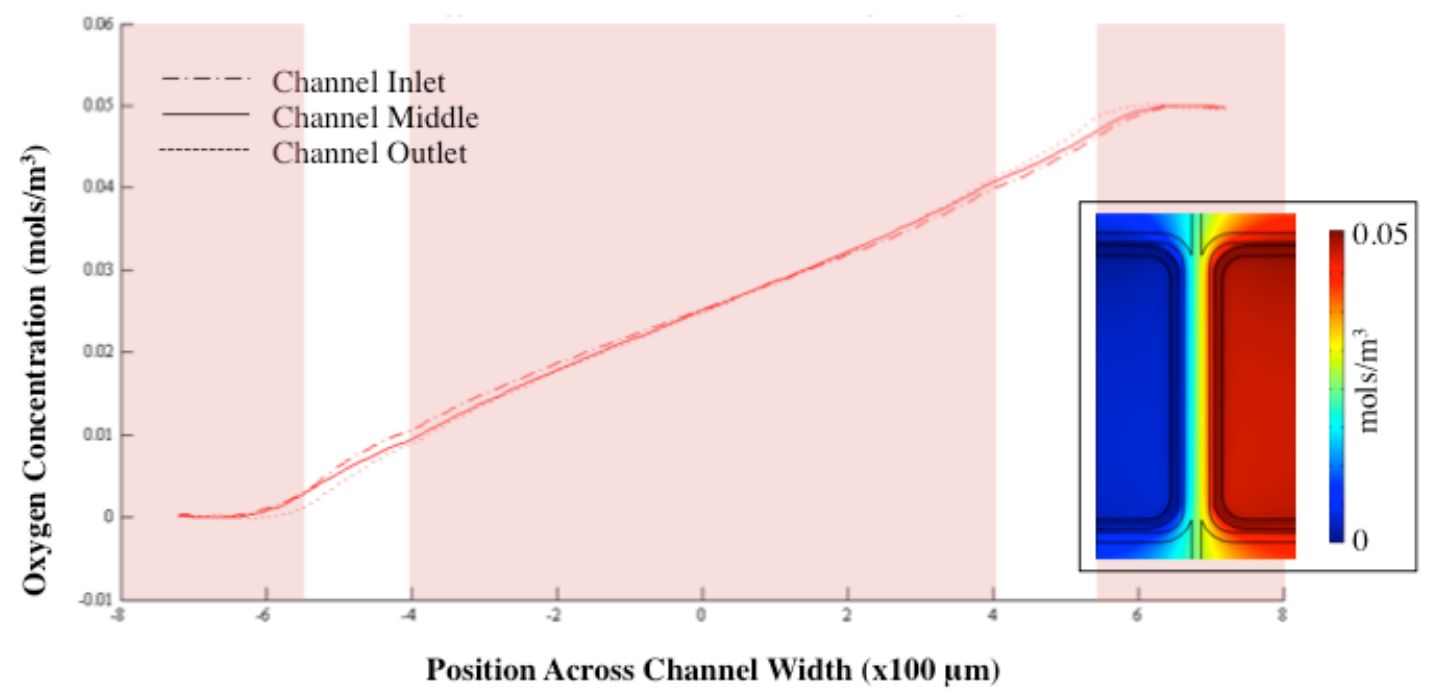

Figure 10. COMSOL ${ }^{\circledR}$-simulated oxygen concentration gradients for the oxygen concentration gradient generator design depicted in Figure 1 but with impermeable outsides of the PDMS block. This simulation was designed to simulate hypoxic oxygen levels in the microchannel (below $10 \mathrm{~mm} \mathrm{Hg}$ ). The inset shows a color map of the oxygen levels at the bottom of the channel. 


\section{CONCLUSIONS}

Here, we have created a time-dependent on-chip drug concentration profile during in vitro testing to mimic a physiological serum concentration. Following drug treatment to induce autophagy, cells showed increased localization of GFP-LC3 and decreased viability. These preliminary results show that the time-dependent environment can induce a different response than the static environment, and we are currently examining this effect.

We have designed an on-chip oxygen gradient generator which uses flow control channels to create a spatial oxygen gradient within a cell culture chamber. This gradient generator was integrated with patterned thin-film optical oxygen sensors. Preliminary results show that using oxygenated and deoxygenated flows within the control channels can create a spatial gradient. Further simulation results show that using an oxygen-impermeable coating on the outside of the microfluidic device will permit the creation of hypoxic environments.

We are also currently investigating the integration of time-varying drug concentration as well as spatial oxygen concentration across a three-dimensional (3D) cell culture model. Microscale 3D constructs can more closely mimic the natural extracellular environment than the two-dimensional cell culture presented here. The tissue culture environment can have a dramatic influence on cell behavior, and this phenomenon has a crucial impact on the responses of cancer cells to therapeutic agents. ${ }^{31}$ Our microfluidic platform will thus feature a 3D hydrogel matrix which can be formed in situ inside the microfluidic platform, encapsulating the cells in well-defined volumes of hydrogels. ${ }^{22,32}$ This $3 \mathrm{D}$ culture system makes it possible to establish growth in multicellular spheroids which mimic solid tumors and will allow us to monitor the response of individual spheroids to drug treatment. The use of the optical luminescence-based oxygen sensor described above permits image overlay to correlate cell viability (as measured using fluorescence microscopy) with oxygen concentrations. With the on-chip oxygen gradient generator, we can also create intermittent hypoxia and examine the effect of intermittent versus chronic hypoxia on cell response.

In future, such platforms, which can be used to culture small numbers of cells and track their response, can provide the ability to analyze small cell populations and assess the drug sensitivity of samples such as primary cancer cells obtained from needle biopsies of solid tumors. These platforms could be a means to assess the impact of treatment standards as well as new treatments designed to achieve more personalized therapy. These platforms will also facilitate cell-based validation in the drug discovery process, decreasing the cost and increasing the speed in screening large numbers of compounds.

\section{ACKNOWLEDGMENTS}

This work was funded in part by the Natural Sciences and Engineering Research Council of Canada (NSERC), the Canadian Institutes of Health Research (CIHR), the Canadian Breast Cancer Research Alliance (CBCRA), CMC Microsystems, and the Canada Foundation for Innovation (CFI). Dr. Marcel Bally (BC Cancer Research Centre) generously provided the MCF7-GFPLC3 cells. Dr. Volker Nock kindly fabricated the oxygen sensors at the University of Canterbury in Christchurch, New Zealand.

\section{REFERENCES}

[1] Keenan, T., and Folch, A., "Biomolecular gradients in cell culture systems," Lab on a Chip, 8(1), 34-57 (2008).

[2] Meyvantsson, I., and Beebe, D. J., "Cell Culture Models in Microfluidic Systems," Annual Review of Analytical Chemistry (2008), 1(1), 423-449 (2008).

[3] Kola, I., and Landis, J., "Can the pharmaceutical industry reduce attrition rates?," Nature Reviews Drug Discovery, 3(8), 711-716 (2004).

[4] Burris, H. A., III, Hurwitz, H. I., Dees, E. C. et al., "Phase I Safety, Pharmacokinetics, and Clinical Activity Study of Lapatinib (GW572016), a Reversible Dual Inhibitor of Epidermal Growth Factor Receptor Tyrosine Kinases, in Heavily Pretreated Patients With Metastatic Carcinomas,” Journal of Clinical Oncology, 23(23), 5305-5313 (2005).

[5] Stoler, D. L., Stewart, C. C., and Stomper, P. C., "Breast Epithelium Procurement from Stereotactic Core Biopsy Washings," Clinical Cancer Research, 8(2), 428-432 (2002). 
[6] Mousa, N. A., Jebrail, M. J., Yang, H. et al., "Droplet-Scale Estrogen Assays in Breast Tissue, Blood, and Serum," Science Translational Medicine, 1(1), 1ra2 (2009).

[7] Klionsky, D. J., and Emr, S. D., "Autophagy as a Regulated Pathway of Cellular Degradation," Science, 290(5497), 1717-1721 (2000).

[8] Kondo, Y., Kanzawa, T., Sawaya, R. et al., "The role of autophagy in cancer development and response to therapy," Nature Reviews Cancer, 5(9), 726-734 (2005).

[9] Tanida, I., Ueno, T., and Kominami, E., "LC3 conjugation system in mammalian autophagy,” The International Journal of Biochemistry \& Cell Biology, 36(12), 2503-2518 (2004).

[10] Kabeya, Y., Mizushima, N., Ueno, T. et al., "LC3, a mammalian homologue of yeast Apg8p, is localized in autophagosome membranes after processing," EMBO J, 19(21), 5720-5728 (2000).

[11] Ogier-Denis, E., and Codogno, P., "Autophagy: a barrier or an adaptive response to cancer," Biochimica et Biophysica Acta (BBA) - Reviews on Cancer, 1603(2), 113-128 (2003).

[12] Vaupel, P., "Tumor microenvironmental physiology and its implications for radiation oncology," Seminars in Radiation Oncology, 14(3), 198-206 (2004).

[13] Harris, A., "Hypoxia - a key regulatory factor in tumour growth," Nature Reviews Cancer, 2(1), 38-47 (2002).

[14] Höckel, M., and Vaupel, P., "Tumor Hypoxia: Definitions and Current Clinical, Biologic, and Molecular Aspects," Journal of the National Cancer Institute, 93(4), 266-276 (2001).

[15] Brown, N., and Bicknell, R., "Hypoxia and oxidative stress in breast cancer: Oxidative stress - its effects on the growth, metastatic potential and response to therapy of breast cancer," Breast Cancer Research, 3(5), 323-327 (2001).

[16] Raleigh, J. A., Dewhirst, M. W., and Thrall, D. E., "Measuring tumor hypoxia," Seminars in Radiation Oncology, 6(1), 37-45 (1996).

[17] Vaupel, P., Briest, S., and Höckel, M., "Hypoxia in Breast Cancer: Pathogenesis, Characterization and Biological/Therapeutic Implications,” Wiener Medizinische Wochenschrift, 152(13-14), 334-342 (2002).

[18] Grist, S. M., Chrostowski, L., and Cheung, K. C., "Optical Oxygen Sensors for Applications in Microfluidic Cell Culture," Sensors, 10(10), 9286-9316 (2010).

[19] Forry, S. P., and Locascio, L. E., "On-chip $\mathrm{CO}_{2}$ control for microfluidic cell culture," Lab on a Chip, 11(23), 4041-4046 (2011).

[20] Desai, S. P., Freeman, D. M., and Voldman, J., "Plastic masters-rigid templates for soft lithography," Lab on a Chip, 9(11), 1631-1637 (2009).

[21] Zhang, L., Yu, J., Pan, H. et al., "Small molecule regulators of autophagy identified by an image-based highthroughput screen," Proceedings of the National Academy of Sciences, 104(48), 19023-19028 (2007).

[22] Chen, M. C. W., Gupta, M., and Cheung, K. C., "Alginate-based microfluidic system for tumor spheroid formation and anticancer agent screening," Biomedical Microdevices, 12(4), 647-654 (2010).

[23] Merkel, T. C., Bondar, V. I., Nagai, K. et al., "Gas sorption, diffusion, and permeation in poly(dimethylsiloxane)," Journal of Polymer Science Part B: Polymer Physics, 38(3), 415-434 (2000).

[24] Ferrell, R. T., and Himmelblau, D. M., "Diffusion coefficients of nitrogen and oxygen in water," Journal of Chemical \& Engineering Data, 12(1), 111-115 (1967).

[25] Tromans, D., "Temperature and pressure dependent solubility of oxygen in water: a thermodynamic analysis," Hydrometallurgy, 48(3), 327-342 (1998).

[26] Nock, V., Alkaisi, M., and Blaikie, R. J., "Photolithographic patterning of polymer-encapsulated optical oxygen sensors," Microelectronic Engineering, 87(5-8), 814-816 (2010).

[27] Bursch, W., Ellinger, A., Kienzl, H. et al., "Active cell death induced by the anti-estrogens tamoxifen and ICI 164384 in human mammary carcinoma cells (MCF-7) in culture: the role of autophagy," Carcinogenesis, 17(8), 1595-1607 (1996).

[28] Qadir, M., Kwok, B., Dragowska, W. et al., "Macroautophagy inhibition sensitizes tamoxifen-resistant breast cancer cells and enhances mitochondrial depolarization," Breast Cancer Research and Treatment, 112(3), 389403 (2008).

[29] Flueckiger, J., Bazargan, V., Stoeber, B. et al., "Characterization of postfabricated parylene C coatings inside PDMS microdevices," Sensors and Actuators B: Chemical, 160(1), 864-874 (2011).

[30] Chaudary, N., and Hill, R. P., "Hypoxia and Metastasis in Breast Cancer," Breast Disease, 26(1), 55-64 (2007).

[31] Abbott, A., "Cell culture: Biology's new dimension," Nature, 424(6951), 870-872 (2003).

[32] Yu, L., Chen, M. C. W., and Cheung, K. C., "Droplet-Based Microfluidic System for Multicellular Tumor Spheroid Formation and Anticancer Drug Testing," Lab on a Chip, 10, 2424-2432 (2010). 